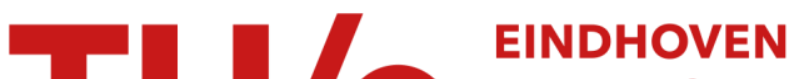 \\ UNIVERSITY OF \\ TECHNOLOGY
}

\section{Rotating ring-ring electrode theory and experiment}

\author{
Citation for published version (APA): \\ Kuiken, H. K., Bakkers, E. P. A. M., Ligthart, H., \& Kellyb, J. J. (2000). Rotating ring-ring electrode theory and \\ experiment. Journal of the Electrochemical Society, 147(3), 1110-1116. https://doi.org/10.1149/1.1393321
}

DOI:

10.1149/1.1393321

Document status and date:

Published: 01/01/2000

\section{Document Version:}

Publisher's PDF, also known as Version of Record (includes final page, issue and volume numbers)

\section{Please check the document version of this publication:}

- A submitted manuscript is the version of the article upon submission and before peer-review. There can be important differences between the submitted version and the official published version of record. People interested in the research are advised to contact the author for the final version of the publication, or visit the $\mathrm{DOI}$ to the publisher's website.

- The final author version and the galley proof are versions of the publication after peer review.

- The final published version features the final layout of the paper including the volume, issue and page numbers.

Link to publication

\section{General rights}

Copyright and moral rights for the publications made accessible in the public portal are retained by the authors and/or other copyright owners and it is a condition of accessing publications that users recognise and abide by the legal requirements associated with these rights.

- Users may download and print one copy of any publication from the public portal for the purpose of private study or research.

- You may not further distribute the material or use it for any profit-making activity or commercial gain

- You may freely distribute the URL identifying the publication in the public portal.

If the publication is distributed under the terms of Article 25fa of the Dutch Copyright Act, indicated by the "Taverne" license above, please follow below link for the End User Agreement:

www.tue.nl/taverne

Take down policy

If you believe that this document breaches copyright please contact us at:

openaccess@tue.nl

providing details and we will investigate your claim. 


\title{
The Rotating Ring-Ring Electrode Theory and Experiment
}

\author{
H. K. Kuiken, ${ }^{\text {a }}$ E. P. A. M. Bakkers, ${ }^{b}$ H. Ligthart, ${ }^{\text {b }}$ and J. J. Kelly ${ }^{b}, *$
}

\author{
${ }^{a}$ Faculty of Applied Mathematics, University of Twente, 7500 AE Enschede, The Netherlands \\ ${ }^{b}$ Debye Institute, Utrecht University, 3508 TA Utrecht, The Netherlands
}

\begin{abstract}
A model is presented for the rotating ring-ring electrode. Although the electrode is defined by four characteristic lengths, it is shown that the collection efficiency depends on only two dimensionless parameters. A simple relationship between these and the corresponding parameters for the rotating ring-disk electrode is pointed out. The model is verified by experiments with the ferricyanide/ferrocyanide system at gold electrodes.
\end{abstract}

(C) 2000 The Electrochemical Society. S0013-4651(99)05-106-X. All rights reserved.

Manuscript submitted May 25, 1999; revised manuscript received November 10, 1999.

The rotating ring-disk electrode (RRDE), first described almost forty years ago by Frumkin and Nekrasov, ${ }^{1}$ has proved an invaluable tool for the study of electrochemical reactions. The RRDE consists of two electrodes in a cylindrical holder: a disk of radius $r_{1}$ (see Fig. 1) surrounded by a concentric ring with inner and outer radii $r_{2}$ and $r_{3}$, respectively. The area between the disk and the ring is electrochemically inactive. The electrodes face downward in solution. The rotation axis passes through the center of the disk $(r=0)$ and is perpendicular to its surface. Products generated at the disk are swept outward by the convection caused by rotation; at the ring they can be detected electrochemically. Using a bipotentiostat one measures a disk current which is generally due to two or more electrochemical reactions. By fixing the potential of the ring it may be possible to distinguish between the products from the disk, i.e., the ring current at a given potential is a measure for the rate of production of a specific product at the disk. In this way complex reactions can be studied both under steady-state and transient conditions., ${ }^{2,3}$

For quantitative analysis the collection efficiency for the RRDE geometry used must be known; this is the fraction of product produced at the disk that is detected at the ring. Early theory of the

* Electrochemical Society Active Member.

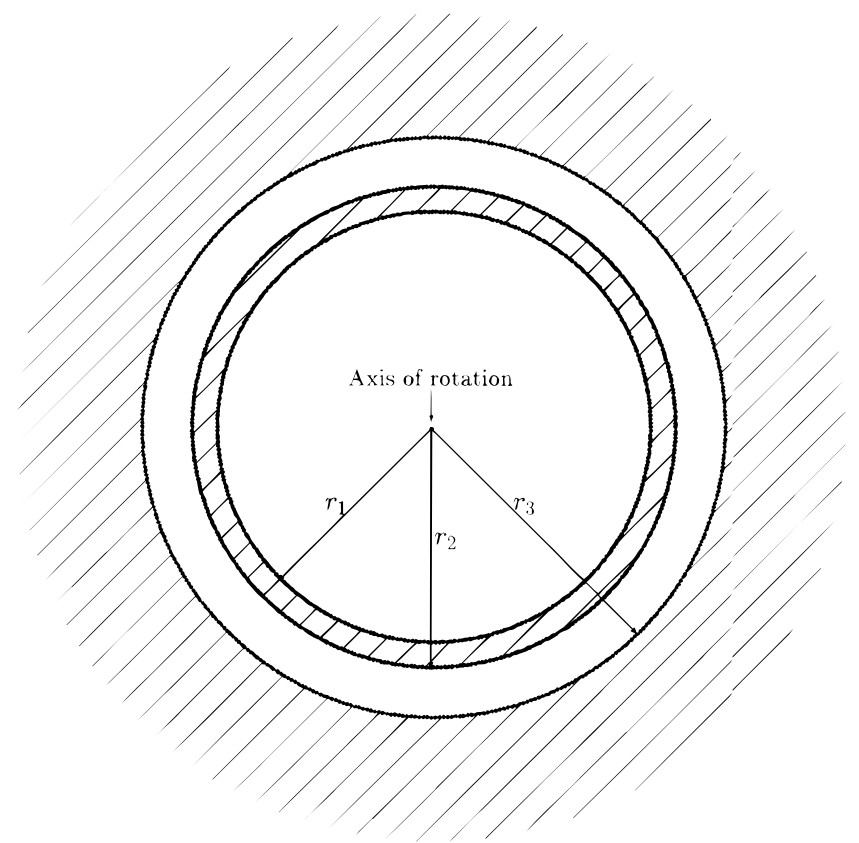

Figure 1. Top view of ring-disk configuration. The nonshaded regions are electrochemically active.
RRDE was developed by Leveque and co-workers. ${ }^{4,5}$ Albery ${ }^{6}$ and Albery and Bruckenstein, ${ }^{7}$ using boundary layer theory and applying the Levèque approximation to describe mass transport, succeeded in setting up a complete and accurate theory for determining the collection efficiency. The theory has been confirmed by experiments in which a single reaction occurs at the disk and its product is measured at the ring. ${ }^{7}$ A survey of work on the RRDE is to be found in books by Albery and Hitchman ${ }^{2}$ and Pleskov and Filinovski. ${ }^{8}$

As part of a project devoted to etching under well-defined hydrodynamic conditions we were interested in etching a ring in a masked rotating disk. In order to follow the dissolution reaction in the ring shaped opening we use an outer concentric ring to detect the etch products. This is, in fact, a rotating ring-ring electrode (RRRE) geometry. To characterize this system we first considered the case in which a simple redox reaction occurs at the inner ring, i.e., no etching occurs. In this paper, we show how the theory of Albery et al. can be extended to cover the RRRE and we derive an expression for the collection efficiency as a function of the ring geometry. Details of the mathematical treatment are presented in a number of Appendices. The experimental setup used to check the validity of the theory is described in the experimental section together with the experimental results.

Unstable products or intermediates formed at the disk of an RRDE can be detected at the ring. ${ }^{2}$ Clearly, the transit time of the species, i.e., the time required for passing from disk to ring, must be shorter than the lifetime of the species. In a separate Appendix, we show that the RRRE can offer advantages for the study of short-lived species. The time required to transport a product to the detecting ring can, for a narrow inner ring, be considerably shorter than for a disk.

The idea of using a rotating double-ring system seems to have been first proposed by Kadija and Nakic ${ }^{9}$ In an earlier paper ${ }^{10}$ they discussed an electrochemical system with gas evolution and they observed that in a classical face-down rotating-disk setup the gas accumulates at the center of the disk because of the low radial velocities there. This motivated them to consider a ring at which the reaction takes place. The radial flow then causes the gas bubbles to move outward. A second outer ring was added to allow detection of reaction products. ${ }^{9}$ Ali and Ambrose ${ }^{11}$ and $\mathrm{Ali}^{12}$ also used a ring-ring electrode configuration in an experimental study of the anodic dissolution of a copper alloy.

Recently, Tolmachev et al., ${ }^{13}$ considered the general mathematical formalism for electrochemical detection systems involving convective flow. They applied their technique to the rotating-ring and the rotating-disk electrode and found good agreement with previous studies. In fact, they observed that their analytical approach yielded better accuracy than digital simulation techniques.

\section{Theory}

The model.-A geometry as depicted in Fig. 2 gives a top view of a disk with two concentric ring-shaped electrochemically active regions which are separated and surrounded by inactive regions. Fig- 


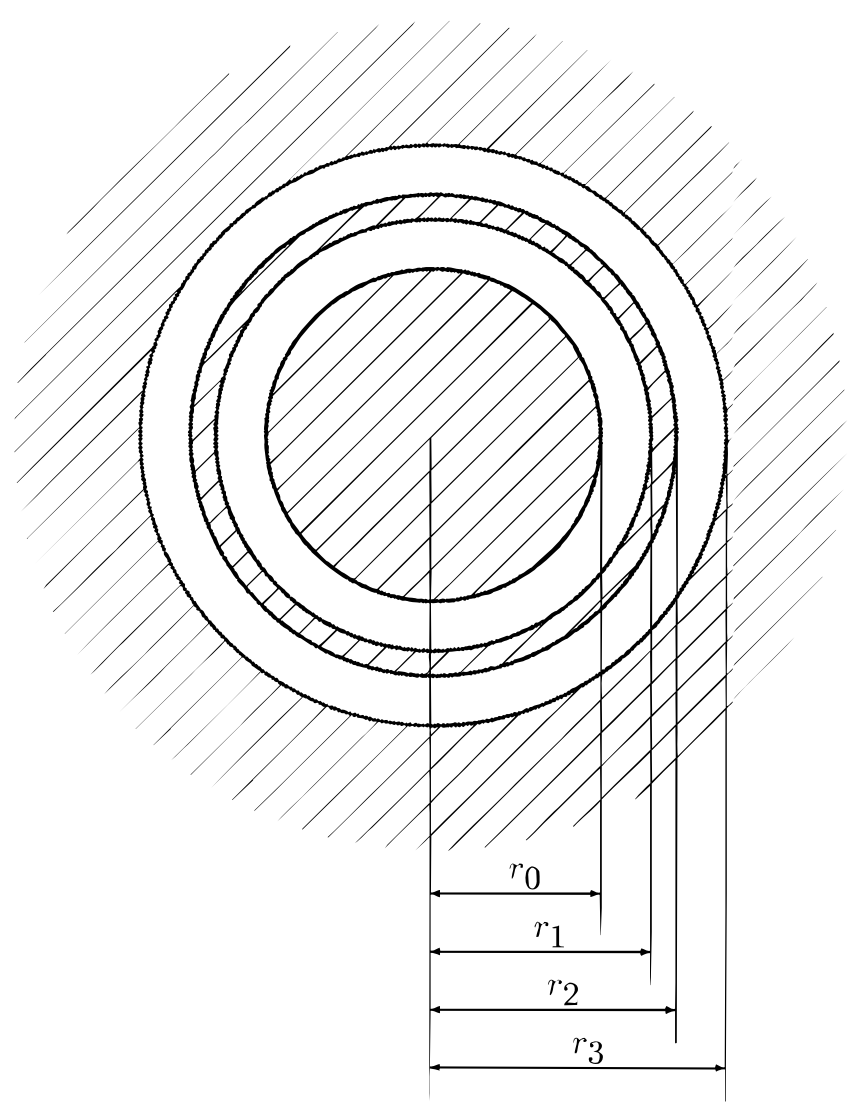

Figure 2. Top view of the ring-ring configuration. The nonshaded rings are electrochemically active.

ure 3 provides a side view of a relevant part of the same system. The inner ring is in the interval $r_{0}<r<r_{1}$ and the outer one is contained in $r_{2}<r<r_{3}$. These are the regions 1 and 3, respectively. The regions 0: $0 \leq r<r_{0}, 2: r_{1}<r<r_{2}$, and 4: $r_{3}<r$ are inactive.

If $c$ is the concentration of an active species which is generated in region 1 by a diffusion-controlled process and we assume that electrochemical detection of this species in region 3 is also diffusion-controlled, then the boundary conditions on the various parts of the disk surface $(y=0)$ are as follows

$$
\begin{gathered}
c=c_{0} \text { for } r_{0}<r<r_{1} \\
c=0 \text { for } r_{2}<r<r_{3} \\
\frac{\partial c}{\partial y}=0 \quad \text { on } \quad 0<r<r_{0}, r_{1}<r<r_{2}, r_{3}<r
\end{gathered}
$$

where a zero gradient of $c$ means that the surface is inactive.

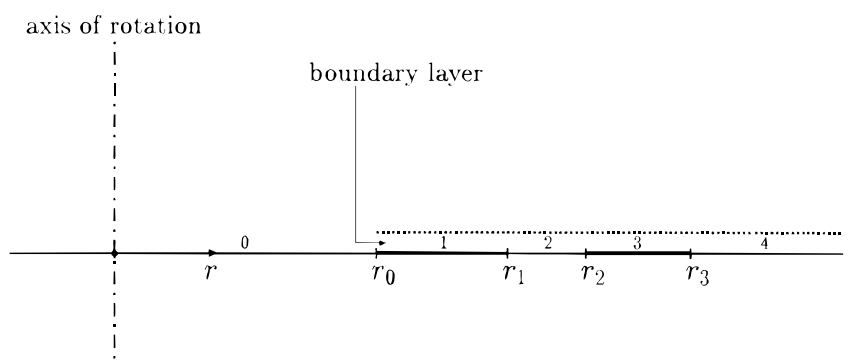

Figure. 3. Side view of the ring-ring configuration. The electrochemically active rings are indicated by 1 and 3, respectively. The concentration boundary layer (not necessarily of uniform thickness) covers the regions 1, 2, 3, and 4. Region 0 is inactive.
To describe mass transport near the surface of the disk, Albery et al. invoke boundary-layer theory. They use the equation

$$
\gamma r y \frac{\partial c}{\partial r}-\gamma y^{2} \frac{\partial c}{\partial y}=D \frac{\partial^{2} c}{\partial y^{2}}
$$

where

$$
\gamma=0.510 \omega^{\frac{3}{2}} v^{-\frac{1}{2}}
$$

Here, $\omega$ is the angular velocity of the disk ( $\mathrm{rads} / \mathrm{s}), v$ is the kinematic viscosity, and $D$ the species diffusivity. Further, $y$ measures normal distance from the disk. It is clear from Eq. 4 that the velocity components in the $r$ and $y$ directions have been approximated by $u$ $=\gamma r y$ and $v=-\gamma y^{2}$, respectively. This is the so-called Levêque approximation which can be used when the concentration boundary layer is much thinner than the hydrodynamic boundary layer. In the present system, this condition is satisfied, since the Schmidt number

$$
S c=\frac{v}{D}
$$

is much larger than unity. Indeed, for diffusion in an aqueous system we have $v \approx 10^{-6} \mathrm{~m}^{2} / \mathrm{s}$ and $D \approx 10^{-10} \mathrm{~m}^{2} / \mathrm{s}$.

The use of boundary-layer theory is permitted when the variation of the concentration in the direction normal to the disk is much faster than along it. In the Conclusion we discuss a typical example showing that this condition is met. Strictly speaking, boundary-layer theory fails close to points where the boundary conditions change abruptly. In the present case, that would mean in regions around the circles $r=r_{\mathrm{i}}$ with $i=0,1,2,3$. As long as the concentration boundary layer is much thinner than the widths of these regions, viz. $r_{1}-$ $r_{0}, r_{2}-r_{1}$, and $r_{3}-r_{2}$, the deviation from boundary-layer theory can be expected to be limited for a global quantity such as a total current or the collection efficiency. However, should the rings become very thin, then it may be necessary to include contributions from nonboundary-layer effects.

To complete the model, we must have a condition defining the concentration field right at the start of the boundary layer, which is at $r=r_{0}$. Since fresh solution enters the boundary layer, we have $c=$ 0 at $r=r_{0}(y \geq 0)$. At the outer edge of the boundary layer the concentration must also approach the value zero.

It should be mentioned that Levich was the first to use transformations of the kind used below or those reported in Ref. 2 and 13. We refer to his book ${ }^{14}$ for the details. In fact, it is mentioned in Ref. 2 that Levich's earliest published results of this kind date back to the early forties.

The three regions. - Region $1\left(r_{0}<r<r_{1}, y \geq 0\right)$.- - Following Albery et al., but using different symbols, we introduce new variables as follows

$$
c=c_{0} \theta(\xi, \eta), \quad \xi=\left(r^{3}-r_{0}^{3}\right) /\left(r_{1}^{3}-r_{0}^{3}\right), \eta=A y r
$$

with

$$
A=\left(\frac{3}{r_{1}^{3}-r_{0}^{3}} \frac{\gamma}{D}\right)^{\frac{1}{3}}
$$

This transforms Eq. 4 into

$$
\eta \frac{\partial \theta}{\partial \xi}=\frac{\partial^{2} \theta}{\partial \eta^{2}}
$$

Since the solution coming in from the direction of the axis through region 0 does not contain the active species, we can prescribe $c=0$ at $r=r_{0}(y>0)$ and thus

$$
\theta=0 \quad \text { at } \quad \xi=0(\eta>0)
$$

Further, Eq. 1 gives

$$
\theta=1 \quad \text { at } \quad \eta=0(\xi>0)
$$


and

$$
\theta \rightarrow 0 \quad \text { if } \quad \eta \rightarrow \infty
$$

The solution to Eq. $9-12$ is

$$
\theta=\Gamma\left(\frac{1}{3}\right)^{-1} \Gamma\left(\frac{1}{3}, \frac{1}{9} \eta^{3} \xi^{-1}\right)
$$

where

$$
\Gamma\left(\frac{1}{3}, z\right)=\int_{z}^{\infty} e^{-\mathrm{p}} p^{-\frac{2}{3}} d p
$$

is an incomplete gamma function and $\Gamma(1 / 3)=\Gamma(1 / 3,0)$.

Since region 1 extends up to $\xi=1$, i.e., $r=r_{1}$, we have from Eq. 7 and 13

$$
c=c_{0} f(\eta)=c_{0} \Gamma\left(\frac{1}{3}\right)^{-1} \Gamma\left(\frac{1}{3}, \frac{1}{9} \eta^{3}\right) \text { at } r=r_{1}
$$

We use this profile as the initial concentration profile for region 2.

Region $2\left(r_{1}<r<r_{2}, y \geq 0\right)$.- -We now write

$$
c=c_{0} \vartheta(\zeta, \mu), \quad \zeta=\left(r^{3}-r_{1}^{3}\right) /\left(r_{2}^{3}-r_{1}^{3}\right), \mu=B y r
$$

with

$$
B=\left(\frac{3}{r_{2}^{3}-r_{1}^{3}} \frac{\gamma}{D}\right)^{\frac{1}{3}}
$$

Since the parameters $A$ (Eq. 8) and $B$ (Eq. 17) are generally different, the boundary-layer variables $\eta$ (Eq. 7, region 1) and $\mu$ (Eq. 16, region 2) are generally different and so are the variables $\xi$ and $\zeta$.

The problem in region 2 is now transformed into

$$
\mu \frac{\partial \vartheta}{\partial \zeta}=\frac{\partial^{2} \vartheta}{\partial \mu^{2}}
$$

with ( $f$ defined by Eq. 15)

$$
\vartheta=f(\kappa \mu) \quad \text { at } \quad \zeta=0(\mu>0)
$$

and (from Eq. 3)

$$
\frac{\partial \vartheta}{\partial \mu}=0 \quad \text { at } \quad \mu=0(\zeta>0)
$$

and

$$
\vartheta \rightarrow 0 \quad \text { as } \quad \mu \rightarrow \infty
$$

The parameter $\kappa$ is given by

$$
\kappa=\left(\frac{r_{2}^{3}-r_{1}^{3}}{r_{1}^{3}-r_{0}^{3}}\right)^{\frac{1}{3}}
$$

For the derivation of the solution

$$
\begin{aligned}
\vartheta(\zeta, \mu)=3^{-\frac{1}{3}} \zeta^{-\frac{2}{3}} e^{-\frac{1}{9} \mu^{3} \zeta^{-1}} \\
\\
\quad \int_{0}^{\infty} q f(\kappa q) e^{-\frac{1}{9} q^{3} \zeta^{-1}} H_{-\frac{1}{3}}\left(\frac{2}{9} \zeta^{-1} \mu^{\frac{3}{2}} q^{\frac{3}{2}}\right) d q
\end{aligned}
$$

and the definition of the function $H$, we refer to Appendix A.

Again, in order to be able to proceed into region 3, this solution must be evaluated at $r=r_{2}$, i.e., at $\zeta=1$

$$
\begin{aligned}
\vartheta(1, \mu)= & g(\mu) \\
& =3^{-\frac{1}{3}} e^{-\frac{1}{9} \mu^{3}} \int_{0}^{\infty} q f(\kappa q) e^{-\frac{1}{9} q^{3}} H_{-\frac{1}{3}}\left(\frac{2}{9} \mu^{\frac{3}{2}} q^{\frac{3}{2}}\right) d q
\end{aligned}
$$

Region 3.- $\left(r_{2}<r<r_{3}, y \geq 0\right)$. - As in the previous regions we now write

$$
c=c_{0} \Theta(\tau, \sigma), \quad \tau=\left(r^{3}-r_{2}^{3}\right) /\left(r_{3}^{3}-r_{2}^{3}\right), \quad \sigma=C y r[25]
$$

with

$$
C=\left(\frac{3}{r_{3}^{3}-r_{2}^{3}} \frac{\gamma}{D}\right)^{\frac{1}{3}}
$$

and our problem is given by

$$
\sigma \frac{\partial \Theta}{\partial \tau}=\frac{\partial^{2} \Theta}{\partial \sigma^{2}}
$$

with

$$
\begin{array}{crrr}
\Theta=g(\lambda \sigma) \quad \text { at } & \tau=0(\sigma>0) \\
\Theta=0 \quad \text { at } & \sigma=0(\tau>0)
\end{array}
$$

and

$$
\Theta \rightarrow 0 \quad \text { as } \quad \sigma \rightarrow \infty
$$

The scaling parameter, $\lambda$, is given by

$$
\lambda=\left(\frac{r_{3}^{3}-r_{2}^{3}}{r_{2}^{3}-r_{1}^{3}}\right)^{\frac{1}{3}}
$$

We again refer to Appendix A for the solution

$$
\begin{array}{r}
\Theta(\tau, \sigma)=3^{-\frac{5}{3}} \tau^{-\frac{4}{3}} e^{-\frac{1}{9} \sigma^{3} \tau^{-1}} \\
\sigma \int_{0}^{\infty} q^{2} g(\lambda q) e^{-\frac{1}{9} q^{3} \tau^{-1}} H_{\frac{1}{3}}\left(\frac{2}{9} \tau^{-1} \sigma^{\frac{3}{2}} q^{\frac{3}{2}}\right) d q
\end{array}
$$

Collection efficiency.-The collection efficiency, $N_{0}$, is defined as the ratio of the amount of the species which is detected in region 3 (outer ring) per unit of time and the total amount of that same species produced in region 1 (inner ring) per unit of time. The first of these terms is defined by

$$
Q_{3}=\left.2 \pi D \int_{r_{2}}^{r_{3}} \frac{\partial c}{\partial y}\right|_{y=0} r d r
$$

and the second by

$$
Q_{1}=-\left.2 \pi D \int_{r_{0}}^{r_{1}} \frac{\partial c}{\partial y}\right|_{y=0} r d r
$$

which gives

$$
N_{0}=Q_{3} / Q_{1}
$$

It is not difficult to show from Eq. 7 and 13 that we may write

$$
Q_{1}=\frac{3^{\frac{1}{3}} \pi}{\Gamma\left(\frac{1}{3}\right)} c_{0} D A\left(r_{1}^{3}-r_{0}^{3}\right)
$$

The derivation of $Q_{3}$ from Eq. 25 and 32 is slightly more complicated (see Appendix B). The result is

$$
Q_{3}=\frac{2}{3} \frac{\pi}{\Gamma\left(\frac{1}{3}\right)} c_{0} D C\left(r_{3}^{3}-r_{2}^{3}\right) \int_{0}^{\infty} q g(\lambda q) \Gamma\left(\frac{1}{3}, \frac{1}{9} q^{3}\right) d q
$$

so that

$$
N_{0}=2 \cdot 3^{-\frac{4}{3}} \kappa^{2} \lambda^{2} \int_{0}^{\infty} q g(\lambda q) \Gamma\left(\frac{1}{3}, \frac{1}{9} q^{3}\right) d q
$$


We show in Appendix $C$ that this expression may be reduced to the computationally simpler form

$$
N_{0}=2 \kappa^{2} \sum_{i=0}^{\infty} \frac{M_{i}(\kappa)}{M_{i}(0)} M_{i}\left(\lambda^{-1}\right)
$$

where

$$
M_{i}(\rho)=\int_{\rho}^{\infty} \frac{d u}{\left(1+u^{3}\right)^{i+1}}
$$

Since

$$
M_{0}(\rho)=\frac{\pi}{2 \sqrt{3}}-\frac{1}{\sqrt{3}} \arctan \left(\frac{2 \rho-1}{\sqrt{3}}\right)-\frac{1}{6} \log \left(\frac{(\rho+1)^{3}}{\rho^{3}+1}\right)
$$

and

$$
M_{i}(\rho)=\frac{3 i-1}{3 i} M_{i-1}(\rho)-\frac{\rho}{3 i\left(1+\rho^{3}\right)^{i}}
$$

the series (Eq. 39) may be computed very efficiently by recursion for any values of $\kappa$ and $\lambda$, i.e., for any particular electrode geometry.

Two remarks are now appropriate. First, the collection efficiency, $N_{0}$ is a function of just two parameters, namely, $\kappa$ and $\lambda$, which involve the geometrical parameters $r_{0}, r_{1}, r_{2}$, and $r_{3}$ only. Albery et al. already proved this for the simpler case where $r_{0}$ is equal to zero. A second observation is that our result (Eq. 39) should agree with that of Albery et al. when $r_{0}$ is equal to zero. Since $r_{0}$ only affects the parameter $\kappa$, our result (Eq. 39) should be the same as that of Albery et al. for all values of $\kappa$ and $\lambda$. By a method which involved an intricate manipulation involving inverse Laplace transforms, Albery et al. established the following result which we recast in terms of our parameters

$$
\begin{aligned}
N_{0}=S\left(\lambda^{-1}\right)+ & \lambda^{2} \kappa^{2} S(\kappa) \\
& -\left(1+\kappa^{3}+\lambda^{3} \kappa^{3}\right)^{\frac{2}{3}} S\left(\lambda^{-1}\left\{1+\kappa^{3}+\lambda^{3} \kappa^{3}\right\}^{\frac{1}{3}}\right)
\end{aligned}
$$

where

$$
S(\rho)=\frac{3^{\frac{3}{2}}}{2 \pi} M_{0}(\rho)
$$

Numerical evaluation shows that Eq. 39 and 43 are indeed equivalent!

From the above, we may conclude that the expression for the collection efficiency of a rotating ring-ring electrode is the same as that obtained years ago for the ring-disk electrode. Although the ringring system has one extra degree of freedom, the collection efficiently is still dependent on only two dimensionless parameters, i.e., if the electrochemical reactions are diffusion-controlled. Albery and Hitchman (Ref. 6, p. 22) use the parameters $\alpha$ and $\beta$ which they defined as

$$
\alpha=\left(r_{2}^{3}-r_{1}^{3}\right) / r_{1}^{3}, \quad \beta=\left(r_{3}^{3}-r_{2}^{3}\right) / r_{1}^{3}
$$

The tabulated results obtained by them carry over to the ring-ring electrode if these parameters are replaced by

$$
\begin{aligned}
& \alpha^{\prime}=\kappa^{3}=\left(r_{2}^{3}-r_{1}^{3}\right) /\left(r_{1}^{3}-\right.\left.r_{0}^{3}\right), \\
& \beta^{\prime}=\lambda^{3} \kappa^{3}=\left(r_{3}^{3}-r_{2}^{3}\right) /\left(r_{1}^{3}-r_{0}^{3}\right)
\end{aligned}
$$

This is a very simple but useful conclusion.

\section{Experimental and Results}

The ring-ring electrodes were made by centering a $0.2 \mathrm{~mm}$ thick gold disk with a diam of $10 \mathrm{~mm}$ on two insulated copper contacts, an inner disk and an outer concentric ring mounted below the face of a cylindrical PVC holder of $10 \mathrm{~mm}$ diam. Silver epoxy was used to ensure good electrical contact between the gold and the copper contacts. The ring-ring geometry in the gold was defined by electrodischarge machining. The copper disk served as contact during machin-
Table I. RRRE and RRDE geometries (radii in $\mathrm{mm}$ ) with calculated and experimentally observed collection efficiencies.

\begin{tabular}{ccccccc} 
& $r_{0}$ & $r_{1}$ & $r_{2}$ & $r_{3}$ & $N_{0}($ cal $)$ & $N_{0}(\exp )$ \\
\hline 1 & 2.67 & 3.33 & 3.67 & 4.33 & 0.358 & 0.354 \\
2 & 3.00 & 3.33 & 3.67 & 5.00 & 0.512 & 0.520 \\
3 & 2.67 & 3.00 & 3.67 & 4.33 & 0.331 & 0.328 \\
4 & 2.90 & 3.10 & 3.65 & 5.00 & 0.488 & 0.483 \\
5 & 0.50 & 2.00 & 2.20 & 3.20 & 0.475 & 0.477 \\
6 & 0.00 & 3.33 & 3.67 & 5.00 & 0.429 & 0.431 \\
7 & 0.00 & 3.00 & 3.67 & 4.33 & 0.286 & 0.266 \\
8 & 0.00 & 3.00 & 3.67 & 5.00 & 0.408 & 0.401
\end{tabular}

ing of the holes in the gold disk. The holes were subsequently filled with Araldite 2020 and the electrode face was polished. The electrode was mounted in a modified Tacussel 101T motor assembly.

Table I gives an overview of the ring-ring electrodes 1-5 used in the present study. In addition, some ring-disk electrodes 6-8 were studied as reference. For the measurements a conventional airtight electrochemical cell was used with a platinum counter electrode and a saturated calomel electrode as reference together with an EG\&G PAR 366A bipotentiostat. Oxygen was purged from the cell by bubbling argon through the solution. The electrolyte was prepared from doubly distilled water and contained $10 \mathrm{mM} \mathrm{K}_{3} \mathrm{Fe}(\mathrm{CN})_{6}$ and $1.0 \mathrm{M}$ $\mathrm{KCl}$. The measurements were performed at room temperature.

Ferricyanide ions were reduced at a diffusion controlled rate at the disk of a RRDE or the inner ring of a RRRE

$$
\mathrm{Fe}(\mathrm{CN})_{6}^{3-}+\mathrm{e}^{-} \rightarrow \mathrm{Fe}(\mathrm{CN})_{6}^{4-}
$$

The rotation rate of the electrode was in the range 1000$3600 \mathrm{rpm}$. The ferrocyanide product was detected at the outer ring by reoxidation, also under mass transport controlled conditions. The collection efficiency was derived from the inner ring or disk current and the outer ring current. Table I shows the results obtained with a series of electrodes. Agreement between observed $\left[N_{0}(\exp )\right]$ and calculated $\left[N_{0}(\mathrm{cal})\right]$ values of the collection efficiency is good in both cases. Clearly, the theory presented here describes satisfactionly the properties of the rotating ring-ring elctrode.

In Appendix D, an expression is derived for the ratio of transit times for the ring-ring $\left(t_{\mathrm{rr}}\right)$ and the ring-disk $\left(t_{\mathrm{rd}}\right)$ electrodes

$$
\frac{t_{\mathrm{rr}}}{t_{\mathrm{rd}}} \approx \frac{d_{1}+2 d_{2}}{r_{0}+r_{1}}
$$

where $d_{1}=r_{1}-r_{0}$ and $d_{2}=r_{2}-r_{1}$ and the radii are given in Fig. 2. In the Appendix, it was pointed out that for the RRDE, $t_{\mathrm{rd}}$ is essentially independent of the area of the disk. It is interesting to calculate the $t_{\mathrm{rr}} / t_{\mathrm{rd}}$ ratio for one of the arbitrary geometries used in Table I. For electrode $2, r_{0}=3.0, r_{1}=3.3$, and $\mathrm{r}_{2}=3.7$; this gives a $t_{\mathrm{rr}} / t_{\mathrm{rd}}$ ratio of 1.1/6.3, which corresponds to a sixfold decrease in transit time. This could be readily improved by increasing $r_{0}$ and $r_{1}$ and decreasing the width of the inner ring and the distance between the rings.

\section{Conclusion}

The theory presented in this paper for the RRRE shows that, although the system has four degrees of freedom (four possible radii), the collection efficiency is determined by just two dimensionless parameters. In addition, the theory gives a result which intuitively might have been expected. The parameters for the two types of electrode (RRDE and RRRE) are equivalent if the disk radius $\left(r_{1}\right)$ of the RRDE is replaced by the inner ring width $\left(r_{1}-r_{0}\right)$ of the RRRE. Experimentally determined values of the collection efficiency of the RRRE agree well with values calculated from the theory. In addition, we show that the use of the RRRE may be advantageous for the study of short-lived intermediates in electrochemical studies.

Earlier in this paper, we remarked that the results obtained here are valid only if boundary-layer theory applies. In a qualitative 
sense, this will mean that the layer through which the concentration varies substantially is thin in comparison with the lengths of the regions under consideration, i.e., the widths of the rings. If this rule is applied to region 1 , which is the first electrochemically active region, then we might say that the boundary layer, if that term applies, at $r=r_{1}$ should be thin in comparision with the ring width $r_{1}-r_{0}$. We estimate the thickness of the boundary layer at $r=r_{1}$ by putting $\eta^{3} / 9=3$ in Eq. 15. At this value, the concentration has dropped to below $10^{-2} c_{0}$ since $f(3) \simeq 0.008$. Equation 7 then shows that the thickness of the concentration layer is given by

$$
y \simeq \frac{3}{A r} \simeq 3\left(\frac{r_{1}-r_{0}}{r_{1}} \frac{D}{\gamma}\right)^{\frac{1}{3}}
$$

where we have restricted ourselves to thin rings for which $r_{1}-r_{0}<<$ $r_{1}$, so that $r_{0} \simeq r_{1} \simeq r$. If $r_{1}-r_{0}$ is of the order of $10^{-4} \mathrm{~m}, r_{1} \sim 5 \times$ $10^{-3} \mathrm{~m}, D \sim 10^{-10} \mathrm{~m}^{2} / \mathrm{s}, \omega \sim 100 \mathrm{rad} / \mathrm{s}$, and $\nu \sim 10^{-6} \mathrm{~m}^{2} / \mathrm{s}$, then Eq. 47 yields a boundary-layer thickness of about $5 \times 10^{-6} \mathrm{~m}$ or $5 \mu \mathrm{m}$. Since the width of the first ring was taken as $100 \mu \mathrm{m}$, boundary-layer theory clearly applies. The ring would need to be very thin indeed or the rotation rate very low for boundary-layer theory to become invalid. A similar conclusion holds for region 3 .

Irrespective of the applicability of boundary-layer theory, the assumptions underlying this theory fail near edges and at locations where boundary conditions change abruptly. Sudden changes in the boundary conditions usually produce singularities in the solution and these in turn are indicative of enhanced local mass transport. Earlier examples of this can be found in diffusion-controlled etching where enhanced etch rates are found near mask edges. ${ }^{15-17}$ The present theory and that of Albery et al. could, therefore, be improved by consideration of such edge effects. Clearly, when the rings become so thin that the boundary layer, while still being relatively thin, becomes thicker than, say, $10 \%$ of the width of the rings, a more refined approach will become useful in the interest of accuracy.

It is interesting to apply our theory to the experimental results reported in Ref. 9. Unfortunately, the accuracy of the ring-diameter measurements reported in Ref. 9 is limited. For instance, Kadija and Nadić give a value of $9.80 \pm 0.10 \mathrm{~mm}$ for the inner radius of the inner ring, i.e., for $r_{0}$. The values for the other radii show a similar spread. We take their average values $r_{0}=9.8, r_{1}=10.08, r_{2}=$ 10.76 , and $r_{3}=11.06$, all in millimeters. If we feed these values into Eq. 46 and then into Eq. 39, a collection efficiency of $N_{0}=0.195$ is found. This compares reasonably well with the measured value of 0.17 reported in Ref. 9 for reactions without gas evolution. If we take the lower bounds of the ring thicknesses considered in Ref. 9, that is $r_{0}=9.8, r_{1}=10.03, r_{2}=10.76$, and $r_{3}=11.01$, then our theory gives $N_{0}=0.173$.

Finally, it seems reasonable to assume that the lack of a proper theory has so far prevented the ring-ring method from becoming a popular tool in electrochemistry. Indeed, very few papers have been devoted to this subject. This is in sharp contrast to the ring-disk method. But then, for this system an adequate theory has been available for a long time. ${ }^{2,6,7}$

\section{Acknowledgment}

The authors gratefully acknowledge financial support from the Dutch Technology Foundation (STW). This research is part of the STW-sponsored project TWI44.3286.

Universiteit Utrecht assisted in meeting the publication costs of this article.

\section{Appendix A}

We consider the following boundary-value problem

$$
\begin{gathered}
\eta \frac{\partial \theta}{\partial \xi}=\frac{\partial^{2} \theta}{\partial \eta^{2}} \quad(0 \leq \xi, 0 \leq \eta) \\
\xi=0(\eta>0): \quad \theta=F(\eta) \\
\eta=0(\xi>0): \quad \theta=0
\end{gathered}
$$

$$
\eta \rightarrow \infty: \quad \theta \rightarrow 0
$$

First, assume that

$$
F(\eta)=\delta(\eta-q)
$$

where $\delta(\cdot)$ is the delta function. Applying the Laplace transform

$$
\bar{\theta}(s, \eta)=\int_{0}^{\infty} e^{-s \xi} \theta(\xi, \eta) d \eta
$$

the problem, Eq. A-1-A-4 may be transformed as follows

$$
\frac{d^{2} \bar{\theta}}{d \eta^{2}}=\eta\{s \bar{\theta}-\delta(\eta-q)\} \quad(\eta \geq 0)
$$

with

$$
\begin{array}{ll}
\eta=0: & \bar{\theta}=0 \\
\eta \rightarrow \infty: & \bar{\theta} \rightarrow \infty
\end{array}
$$

Since $\delta(\eta-q)$ is equal to zero for any $\eta \neq q$, Eq. A-7 can essentially be replaced by

$$
\frac{d^{2} \bar{\theta}}{d \eta^{2}}=s \eta \bar{\theta}
$$

The influence of the delta function is then obtained from an integration of Eq. A-8 from $\eta=q-0$ to $\eta=q+0$. This gives

$$
\left.\frac{\partial \bar{\theta}}{\partial \eta}\right|_{\eta=\mathrm{q}-0} ^{\eta=\mathrm{q}+0}=-q
$$

showing that the derivative experiences a jump at $\eta=q$.

The solution of Eq. A-10 which is continuous at $\eta=q$ and satisfies the conditions of Eq. A-3, A-4, and A-11 is

$$
\begin{gathered}
\bar{\theta}=\frac{2}{3} \eta^{\frac{1}{2}} q^{\frac{3}{2}} K_{\frac{1}{3}}\left(\frac{2}{3} s^{\frac{1}{2}} q^{\frac{3}{2}}\right) I_{\frac{1}{3}}\left(\frac{2}{3} s^{\frac{1}{2}} \eta^{\frac{3}{2}}\right) \quad(0 \leq \eta<q) \\
\bar{\theta}=\frac{2}{3} \eta^{\frac{1}{2}} q^{\frac{3}{2}} I_{\frac{1}{3}}\left(\frac{2}{3} s^{\frac{1}{2}} q^{\frac{3}{2}}\right) K_{\frac{1}{3}}\left(\frac{2}{3} s^{\frac{1}{2}} \eta^{\frac{3}{2}}\right) \quad(\eta>q)
\end{gathered}
$$

where $I_{1 / 3}(\cdot)$ and $K_{1 / 3}(\cdot)$ are modified Bessel functions. We refer to Ref. 18, pp. 446-447, for the details.

The Laplace inverses of Eq. A-12 and A-13 can be found in Ref. 19, formula 13.96, p. 346. These yield

$$
\theta(\xi, \eta)=\frac{1}{3} \xi^{-1} \eta^{\frac{1}{2}} q^{\frac{3}{2}} \exp \left(-\frac{\eta^{3}+q^{3}}{9 \xi}\right) I_{\frac{1}{3}}\left(\frac{2}{9} \xi^{-1} \eta^{\frac{2}{3}} q^{\frac{3}{2}}\right)
$$

It is computationally and conceptually simpler to use

$$
I_{v}(z)=\left(\frac{1}{2} z\right)^{v} H_{v}(z)
$$

where

$$
H_{v}(z)=\sum_{i=0}^{\infty} \frac{\left(\frac{1}{4} z^{2}\right)^{\mathrm{i}}}{i ! \Gamma(v+i+1)}
$$

Using convolution, we can write the solution to the full problem, Eq. A-1-A-4, as

$$
\theta(\xi, \eta)=3^{-\frac{5}{3}} \xi^{\frac{4}{3}} \eta \int_{0}^{\infty} q^{2} F(q) \exp \left(-\frac{\eta^{3}+q^{3}}{9 \xi}\right) H_{\frac{1}{3}}\left(\frac{2}{9} \xi^{-1} \eta^{\frac{3}{2}} q^{\frac{3}{2}}\right) d q
$$

We may now define a second problem which is obtained from Eq. A-1-A-4 by replacing Eq. A-3 by

$$
\eta=0(\xi>0): \quad \frac{\partial \theta}{\partial \eta}=0
$$

The solution procedure is similar to that of the previous problem and the result is that given by Eq. 23 with $f(\kappa q)$ replaced by $F(q)$. 


\section{Appendix B}

The mass flow through region 1 as defined by Eq. 34 involves the integral

$$
\left.\int_{r_{0}}^{r_{1}} \frac{\partial c}{\partial y}\right|_{y=0} r d r
$$

where $c$ is given by Eq. 7 and 13. The integral may, therefore, be written as

$$
\left.\frac{1}{3} c_{0} A\left(r_{1}^{3}-r_{0}^{3}\right) \int_{0}^{1} \frac{\partial \theta}{\partial \eta}\right|_{\eta=0} d \xi
$$

Calculating the derivative from Eq. 13 and 14, we may replace Eq. B-2 by

$$
3^{-\frac{2}{3}} \Gamma\left(\frac{1}{3}\right)^{-1} c_{0} A\left(r_{1}^{3}-r_{0}^{3}\right) \int_{0}^{1} \xi^{-\frac{1}{3}} d \xi
$$

and the result given by Eq. 36 follows easily.

Regarding Eq. 33, note that by using Eq. 25, 32, and A-15, we may write

$$
\begin{aligned}
& \left.\int_{r_{2}}^{r_{3}} \frac{\partial c}{\partial y}\right|_{y=0} r d r=\left.\frac{1}{3} c_{0} C\left(r_{3}^{3}-r_{2}^{3}\right) \int_{0}^{1} \frac{\partial \Theta}{\partial \sigma}\right|_{\sigma=0} d \tau \\
& \quad=3^{-\frac{5}{3}} \Gamma\left(\frac{1}{3}\right)^{-1} c_{0} C\left(r_{3}^{3}-r_{2}^{3}\right) \int_{0}^{\infty} q^{2} g(\lambda q)\left[\int_{0}^{1} \tau^{-\frac{4}{3}} \mathrm{e}^{-\frac{1}{9} \tau^{-1} q^{3}} d \tau\right] d q
\end{aligned}
$$

Since

$$
\int_{0}^{1} \tau^{-\frac{4}{3}} \mathrm{e}^{-\frac{1}{9} \tau^{-1} q^{3}} d \tau=3^{\frac{2}{3}} q^{-1} \Gamma\left(\frac{1}{3}, \frac{1}{9} q^{3}\right)
$$

the result, Eq. 37, follows easily.

\section{Appendix C}

The reduction of Eq. 38 to 39 runs as follows. Substituting the function $f$ given by Eq. 15 in Eq. 24 we have

$$
g(q)=3^{-\frac{1}{3}} \Gamma\left(\frac{1}{3}\right)^{-1} \mathrm{e}^{-\frac{1}{9} \mathrm{q}^{3}} \int_{0}^{\infty} p \Gamma\left(\frac{1}{3}, \frac{1}{9} \kappa^{3} p^{3}\right) \mathrm{e}^{-\frac{1}{9} \mathrm{p}^{3}} H_{-\frac{1}{3}}\left(\frac{2}{9} p^{\frac{3}{2}} q^{\frac{3}{2}}\right) d p
$$

Substituting this in turn in Eq. 38 a double integral representing $N_{0}$ is obtained. If we now use the series representation of $H_{-1 / 3}(\cdot)$ as given by Eq. A-15, this double integral may be written as an infinite sum of products of separated integrals

$$
N_{0}=\frac{2 \cdot 3^{-\frac{5}{3}}}{\Gamma\left(\frac{1}{3}\right)} \kappa^{2} \lambda^{2} \sum_{i=0}^{\infty} \frac{\lambda^{3 i}}{3^{4 i} i ! \Gamma\left(i+\frac{2}{3}\right)} L_{i}(1, \lambda) L_{i}(\kappa, 1)
$$

where

$$
L_{\mathrm{i}}(a, b)=\int_{0}^{\infty} \rho^{3 i+1} \Gamma\left(\frac{1}{3}, \frac{1}{9} a^{3} \rho^{3}\right) \mathrm{e}^{-\frac{1}{9} \mathrm{~b}^{3} \rho^{3}} d \rho
$$

We can reduce this integral to a simpler form by differentiating it with respect to $a$ and then integrating the resulting integral. This gives

$$
\frac{\partial L_{i}(a, b)}{\partial a}=-\frac{3^{\frac{4}{3}+2 i} i !}{\left(a^{3}+b^{3}\right)^{i+1}}
$$

Since $L_{\mathrm{i}}(a, b)$ tends to zero when $a \rightarrow \infty$, then

$$
L_{i}(a, b)=3^{\frac{4}{3}+2 i} i ! b^{-2-3 i} M_{i}\left(\frac{a}{b}\right)
$$

where $M_{i}(\rho)$ is given by Eq. 40 . Equation C-2 can now be written as follows

$$
N_{0}=\frac{6}{\Gamma\left(\frac{1}{3}\right)} \kappa^{2} \sum_{i=0}^{n} \frac{i !}{\Gamma\left(i+\frac{2}{3}\right)} M_{\mathrm{i}}(\kappa) M_{i}\left(\frac{1}{\lambda}\right)
$$

If we observe that

$$
M_{i}(0)=\frac{1}{3} \frac{\Gamma\left(\frac{1}{3}\right) \Gamma\left(i+\frac{2}{3}\right)}{i !}
$$

then Eq. 39 follows immediately.

\section{Appendix D}

Estimate of the transit time of a particle residing in the flow induced by a rotating disk.-

Ring-disk.-

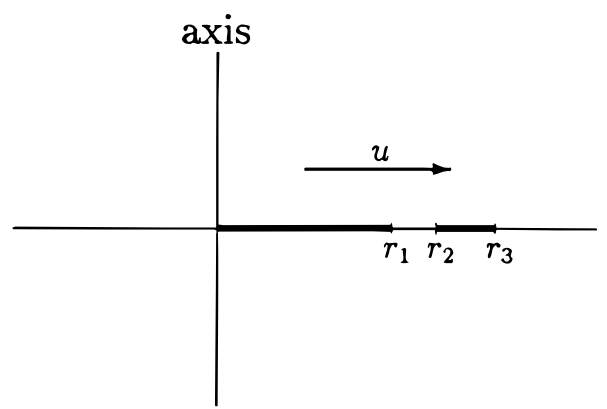

Figure D-1. Ring-disk geometry.

The maximum value of $u$ is

$$
u \sim c_{1} \Omega r
$$

where $r$ is the distance from the axis; $\Omega$ is the angular velocity of the disk and $c_{1}$ is a constant that is approximately equal to 0.18 . From $u=d r / d t$ we have

$$
d t=\frac{1}{c_{1} \Omega} \frac{d r}{r}
$$

The time for a particle to move from $r=a$, with $0<\mathrm{r}<a$, to $r=r_{2}$ can be derived from Eq. D-2 as follows

$$
t_{\mathrm{a}}=\frac{1}{c_{1} \Omega}\left(\log r_{2}-\log a\right)
$$

Then, the average time for all particles originating on the disk $\left(0<r<r_{1}\right)$ is given by

$$
t_{\mathrm{rd}}=\frac{2 \pi \int_{0}^{\eta_{1}} t_{\mathrm{a}} a d a}{\pi r_{1}^{2}}
$$

or

$$
t_{\mathrm{rd}}=\frac{1}{c_{1} \Omega r_{1}^{2}} \int_{0}^{\eta_{1}}\left(\log r_{2}-\log a\right) d a^{2}=\frac{1}{c_{1} \Omega}\left(\frac{1}{2}+\log \frac{r_{2}}{r_{1}}\right)
$$

Since $r_{1} \approx r_{2}$, it follows that the average transit time is almost independent of the size of the disk. Since

$$
\frac{r_{2}-r_{1}}{r_{1}} \ll<1
$$

we have

$$
t_{\mathrm{rd}} \sim \frac{1}{c_{1} \Omega}\left(\frac{1}{2}+\frac{r_{2}-r_{1}}{r_{1}}\right) \geq \frac{1}{2} \frac{1}{c_{1} \Omega}
$$

Ring-ring.-

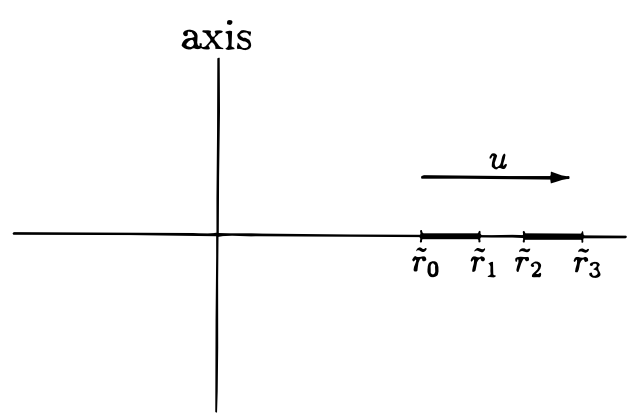

Figure D-2. Ring-ring geometry. 
We must now look at the average transit time of particles originating on the inner ring $\left(\tilde{r}_{0}<r<\tilde{r}_{1}\right)$. We have from Eq. D-3

$$
t_{\mathrm{rr}}=\frac{\frac{1}{c_{1} \Omega} \int_{\tilde{r}_{0}}^{\tilde{r}_{1}}\left(\log \tilde{r}_{2}-\log a\right) 2 \pi a d a}{\pi\left(\tilde{r}_{1}^{2}-\tilde{r}_{0}^{2}\right)}
$$

$$
t_{\mathrm{rr}}=\frac{1}{c_{1} \Omega}\left(\frac{1}{2}+\frac{\tilde{r}_{1}^{2} \log \frac{\tilde{r}_{2}}{\tilde{r}_{1}}-\tilde{r}_{0}^{2} \log \frac{\tilde{r}_{2}}{\tilde{r}_{0}}}{\tilde{r}_{1}^{2}-\tilde{r}_{0}^{2}}\right)
$$

Again, we have

$$
\frac{\tilde{r}_{2}-\tilde{r}_{1}}{\tilde{r}_{1}} \ll<1 \text { and } \frac{\tilde{r}_{2}-\tilde{r}_{0}}{\tilde{r}_{0}} \ll 1
$$

so that

$$
t_{\mathrm{rr}} \sim \frac{1}{c_{1} \Omega}\left(-\frac{1}{2}+\frac{\tilde{r}_{2}}{\tilde{r}_{0}+\tilde{r}_{1}}\right)=\frac{1}{c_{1} \Omega} \frac{\frac{1}{2} d_{1}+d_{2}}{\tilde{r}_{0}+\tilde{r}_{1}}
$$

where $d_{1}=\widetilde{r}_{1}-\widetilde{r}_{0}$ is the width of the inner ring and $d_{2}=\widetilde{r}_{2}-\widetilde{r}_{1}$ is the distance between the rings. We have from Eq. D-6 and D-9

$$
\frac{t_{\mathrm{rr}}}{t_{\mathrm{rd}}} \sim \frac{d_{1}+2 d_{2}}{\tilde{r}_{0}+\tilde{r}_{1}}
$$

Usually, the radii of the circular edges will be chosen such that the following condition is satisfied

$$
\frac{d_{1}+2 d_{2}}{\tilde{r}_{0}+\tilde{r}_{1}} \ll 1
$$

Therefore

$$
t_{\mathrm{rr}}<<t_{\mathrm{rd}}
$$

\section{References}

1. A. N. Frumkin and L. N. Nekrasov, Dokl. Akad. Nauk SSSR, 126, 115 (1959).

2. W. J. Albery and M. L. Hitchman, Ring-Disc Electrodes, Clarendon Press, Oxford (1971)

3. G. H. Schoenmakers, R. Waagenaar, and J. J. Kelly, Ber. Bunsen-Ges. Phys. Chem., 100, 116 (1996)

4. Yu. V. Ivanov and V. G. Levich, Dokl. Akad. Nauk SSSR, 126, 1029 (1959).

5. A. N. Frumkin, L. N. Nekrasov, B. G. Levich, and Yu. B. Ivanov, J. Electroanal. Chem., 1, 84 (1959).

6. W. J. Albery, Trans. Faraday Soc., 62, 1915 (1966).

7. W. J. Albery and S. Bruckenstein, Trans. Faraday Soc., 62, 1920 (1966).

8. Yu. V. Pleskov and V. Yu. Filinovskii, The Rotating Disk Electrode, Consultants Bureau, New York (1976).

9. I. V. Kadija and V. M. Nakić, Electroanal. Chem. and Interfacial Electrochem., 35, 177 (1972).

10. I. V. Kadija and V. M. Nakić, Electroanal. Chem. and Interfacial Electrochem., $\mathbf{3 4}$ 15 (1972).

11. J. A. Ali and J. R. Ambrose, Corros. Sci., 32, 799 (1991).

12. J. A. Ali, Corros. Sci., 36, 773 (1994).

13. Y. V. Tolmachev, Z. Wang, and D. A. Scherson, J. Electrochem. Soc., 143, 3539 (1996).

14. B. Levich, Physicochemical Hydrodynamics, Prentice-Hall, Englewood Cliffs, NJ (1962).

15. H. K. Kuiken, Proc. R. Soc. London, A392, 199 (1984)

16. H. K. Kuiken, J. J. Kelly, and P. H. L. Notten, J. Electrochem. Soc.. 133, 1217 (1986).

17. P. H. L. Notten, J. J. Kelly, and H. K. Kuiken, J. Electrochem. Soc., 133, 1226 (1986).

18. M. Abramowitz and I. A. Stegun, Handbook of Mathematical Functions, 7th printing, National Bureau of Standards, Washington, DC (1964).

19. F. Oberhettinger and L. Badii, Tables of Laplace Transforms, Springer, Berlin (1973). 